AIAA-2002-0879

\title{
RESULTS FROM A STING WHIP CORRECTION VERIFICATION TEST AT THE LANGLEY 16-FOOT TRANSONIC TUNNEL
}

\author{
B. L. Crawford* \\ T. D. Finley ${ }^{\star}$ \\ NASA Langley Research Center \\ Hampton, VA 23681-2199
}

\begin{abstract}
In recent years, great strides have been made toward correcting the largest error in inertial Angle of Attack (AoA) measurements in wind tunnel models. This error source is commonly referred to as "sting whip" and is caused by aerodynamically induced forces imparting dynamics on sting-mounted models. These aerodynamic forces cause the model to whip through an arc section in the pitch and/or yaw planes, thus generating a centrifugal acceleration and creating a bias error in the AoA measurement. It has been shown that, under certain conditions, this induced AoA error can be greater than one third of a degree. An error of this magnitude far exceeds the target AoA goal of $0.01^{\circ}$ established at NASA Langley Research Center (LaRC) and elsewhere. New sting whip correction techniques being developed at LaRC are able to measure and reduce this sting whip error by an order of magnitude. With this increase of accuracy, the $0.01^{\circ}$ AoA target is achievable under all but the most severe conditions.
\end{abstract}

Until recently, LaRC has not had the opportunity to independently verify the validity of these sting whip correction systems under wind tunnel test conditions. In January 2001 , a testing opportunity presented itself at the Langley 16-Foot Transonic Tunnel (16-FT TT) where two video photogrammetric systems and an arcsector AoA sensor corrected for sting deflections were available for verification. These systems are not affected

\footnotetext{
"Electronics Research Engineer, Member AIAA
}

${ }^{\dagger}$ Senior Research Engineer

Copyright $(\mathcal{C} 2002$ by the American Institute of Aeronautics and Astronautics, Inc. No copyright is asserted in the United States under Title 17, U.S. Code. The U.S. Government has a royalty-free license to exercise all rights under the copyright claimed herein for Governmental Purposes. All other rights are reserved by the copyright owner. by model dynamics. Additionally, there were two sting whip systems employing two different correction techniques mounted in the model at different dynamic radii. This configuration allowed for four comparisons to either sting whip system.

The same acceleration that causes an error in the AoA measurement also acts on the model and induces an error in the balance axial force reading. An extrapolation equation was derived that uses the measured acceleration generated by a sting whip correction system to scale the correction to any location along the model's length. This enables the user to correct for errors in the balance reading or errors induced in any other acceleration sensitive device. Having two sting whip systems in this test supplied a means to check the extrapolation technique. This technique has been successfully demonstrated in the lab but did not compare favorably with the sting whip measurements in the first tunnel test.

In getting to this degree of accuracy, LaRC researchers have been through several iterations of sting whip system configurations over the past three years. The process involved several arrangements of sensors, $\mathrm{AD} / \mathrm{DA}$ boards, filtering techniques, software versions and computer systems with each configuration having its merits with regard to accuracy, cost, reliability, and size. As this research effort comes to an end, the following paper will discuss in detail the verification setup, test and results, and the concept of being able to correct for centrifugal accelerations anywhere along the model axis and latest version of the sting whip system.

\section{Test Objectives}

The tunnel time to run this test was donated to the sting whip cause by the management of the Langley 16-Foot Transonic Tunnel. Hence, this was a stand-alone test and not piggy-backed on another test. With this in mind we were able to set the test 
parameters to further validation of the sting whip correcting AoA measurement system.

The first objective was to verify the accuracy of the sting whip correcting AoA measurement system with an independent device. The second objective was to verify that the extrapolation technique predicts centrifugal acceleration at any other point along the model axis.

\section{Test Setup}

The test occurred in the Langley 16-Foot Transonic Tunnel. This tunnel is a closed loop system capable of transonic speeds up to mach 1.2. The test section has an octagonal cross section roughly $16 \mathrm{ft}$ in "diameter."

A high-speed research (HSR) model was used for the test. It was mounted on a sting arc sector configuration with remotely controlled pitch capabilities. The test plan called for a pitch range from $-4^{\circ}$ to $10^{\circ}$ in two degree increments set randomly to help guard against systematic errors. The speed ranges were set at $M=0.0,0.3,0.8$, and 0.9 to give a varying degree of sting whip error. The random polars were run at each mach number and repeated three times with the $M=0.0$ polar occurring at the start and end of the test and after each mach number set. The first and last windoff polars were performed with the additional use of the AoA "reference" angle measurement system (AMS) attached. This facilitated the calibration of the AoA systems and allowed for corrections of any bias shift that may have occurred during the test. This test configuration would yield a wide range of dynamic conditions to investigate.

There were five AoA devices used in the test. Two were video model deformation (VMD) systems with AoA measurement capabilities.' One was a standard
LaRC inertial AoA sensor mounted in the strut and corrected with calculated sting deflections based on balance output. The other two systems were sting whip correction systems referred to as QS! and QS2.

The VMD systems were located outside of the test section and plenum, and looked through a window at a series of $1 / 2$ in. diameter retro reflective targets located on the side of the fuselage. QS2 and QS1 were located inside the fuselage $11.983 \mathrm{in}$. and $24.379 \mathrm{in}$. respectively in front of the model $C G$.

\section{Strut Mounted AoA Corrected For Sting Deflections}

The strut mounted AoA corrected for the sting deflections method of measuring $A O A$ was convenient due to infrastructure being already in place (inertial accelerometer in strut and equations in DAS), but was not necessarily an accurate method. One possible explanation for the inaccuracy is that the sting deflections are based entirely on balance output. ${ }^{2}$ This only takes into account the forces acting on the model and not on the sting itself. A preliminary investigation into the forces acting on the sting and the resulting deflections was performed. This analysis showed that there can be significant sting deflections due to forces acting on the model support system, but does not account for all of the inaccuracy. Therefore, we left this AoA estimate out of our best extimate of AoA. To establish our best estimate of AoA, we averaged the two VMD estimates with the two sting whip estimates. Then, subtracting that from the sting deflection estimate, there is a big discrepancy. This discrepancy is depicted along with the predicted amount of sting deflection due to the wind impinging on the sting in Figure 1 .

As a result of this phenomenon, the sting deflection estimate of AOA was not included as part of the best estimate for the AoA reading.

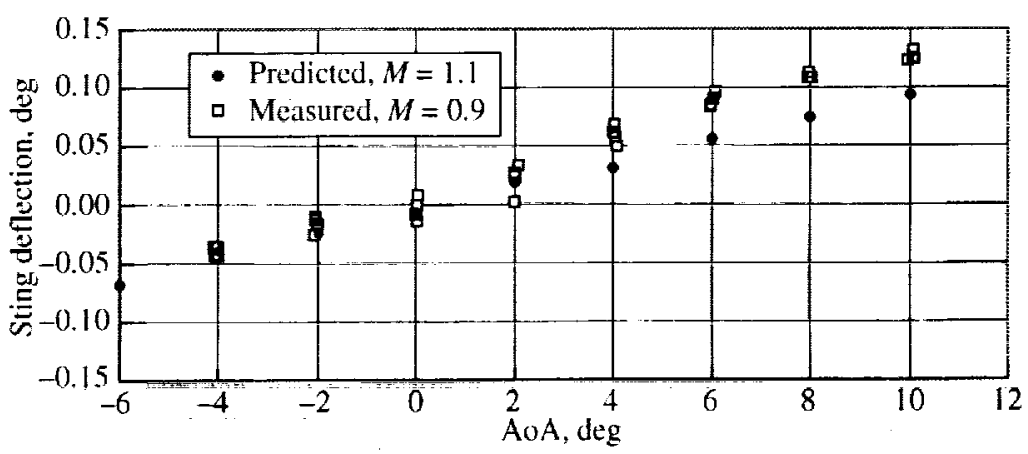

Figure 1. Sting deflection due to wind-on sting. 


\section{Equations}

\section{Sting Whip Correction Equations:}

The equations are presented below in both the corrected and uncorrected form. In doing so the sting whip correction amount can be established by taking the difference between the corrected and uncorrected values.

The uncorrected equation, shown as eq. 1 , is the standard form that has been used at LaRC for many years.

$$
\mathrm{QSI}_{u}=a \sin \left(\frac{V_{\mathrm{qs} 1}-B_{\mathrm{qs} 1}}{S_{\mathrm{qs} 1}}\right)-\phi
$$

QS1 AoA corrected for sting whip is:

$$
\operatorname{Pitch}_{q \times 1}=a \sin \left[\left(\frac{V_{\mathrm{q} 1}-B_{\mathrm{qsi}}}{S_{q \times 1}}\right)-\frac{C(V)}{573} *\left(1-\frac{t-75}{562}\right)\right]-\phi
$$

The uncorrected QS2 is handled in the same way as the QSI.

$$
\mathrm{QS} 2_{u}=a \sin \left(\frac{V_{\mathrm{qs} 2}-B_{\mathrm{qs} 2}}{S_{\mathrm{qs} 2}}\right)-\phi
$$

The QS2 correction differs from QS1 in the correction term. It is in terms of yaw and pitch g's.

$$
G_{y / p}=\frac{V_{y / p}}{572.96}\left(1+\frac{M_{y / p}}{100}(T-75)\right)
$$

Once the g's have been measured, they can be put into the QS2 equation to obtain the AoA measurement corrected for sting whip.

$$
\operatorname{Pitch}_{\mathrm{qs} 2}=a \sin \left(\frac{V_{\mathrm{q} \times 2}-B_{\mathrm{qs} 2}}{S_{\mathrm{qs} 2}}-\left(G_{y}+G_{p}\right)\right)-\phi
$$

where:

QS $1_{\mathrm{u}} / \mathrm{QS2}=\mathrm{QS1}$, QS2 AoA uncorrected

$V_{\mathrm{qs} 1} / V_{\mathrm{q} s 2}=\mathrm{QS} 1$, QS2 AoA sensor voltage

$B_{\mathrm{qs} 1} / B_{\mathrm{q} 2} 2=\mathrm{QS} 1, \mathrm{QS} 2$ AoA sensor bias

$S_{\mathrm{q}: 1} / S_{\mathrm{qs} 3}=\mathrm{QS} 1, \mathrm{QS} 2$ AoA sensor sensitivity

$\phi=$ QS1, QS2 AoA sensor offset

$\mathrm{Pitch}_{\mathrm{qs} 1} / \mathrm{Pitch}_{\mathrm{qs} 2}=$ QS1 AoA sting whip corrected

$C(V)=$ correction voltage

$t=$ temperature sensor
Acceleration Extrapolation:

In 1969, Dr. Frank W. Steinle Jr. conducted a test that demonstrated the existence of a centrifugal acceleration acting on a sting-mounted model. ${ }^{3}$ He concluded that "random motion of a sting-model support system can induce a thrusting bias error to axial force, and hence drag, measurement." The same centrifugal acceleration experienced by the balance axial also influences the inertial AoA accelerometer. This acceleration can be measured and corrected for at the AoA sensor and can also be extrapolated to correct for the acceleration acting on a secondary accelerometer or even the balance axial output. The extrapolation equation can be derived easily using Figure 2 .

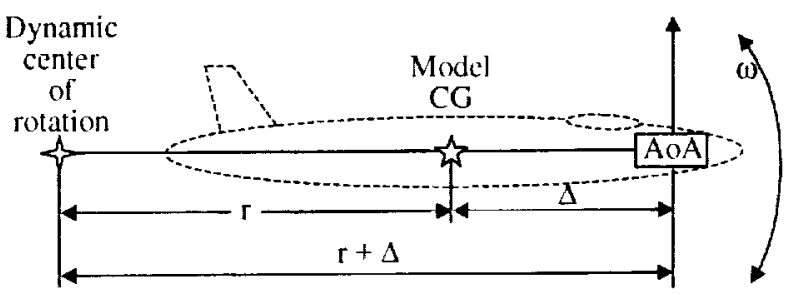

Figure 2. Extrapolation equation model.

From this figure the centrifugal acceleration at the model center of gravity (CG) is calculated using:

$$
a_{c g}=\omega^{2} r
$$

where:

$\omega=$ angular rate

$r=$ radius

The centrifugal acceleration at the AoA sensor is:

$$
a_{\mathrm{AoA}}=\omega^{2}(r+\Delta)=\omega V
$$

Since the $r$ term is difficult to measure, we take equation 7 and solve for $r$ to get:

$$
r=\frac{V-\omega \Delta}{\omega}=\frac{V}{\omega}-\Delta
$$

Then, substituting the solution from equation 8 into equation 6 for $r$ we get the acceleration in terms that are much easier to determine.

$$
a_{c g}=\omega^{2}\left(\frac{V}{\omega}-\Delta\right)
$$


Simplifying equation 9 gives us an equation in the form of a straight line.

$$
a_{c g}=\omega V-\omega^{2} \Delta
$$

Where $\omega V$ is a known acceleration point, $\omega^{2}$ is the slope value for the acceleration and $\Delta$ is the distance along the model axis from the AoA sensor. After applying conversion and scaling factors to the output of the sensors, equation 10 becomes:

$$
a_{c g}=\frac{\operatorname{Cor}(V)}{573}+\frac{\operatorname{Slope}(V)}{1932} \Delta
$$

Here the $\operatorname{Corr}(V)$ is the correction voltage at the $A \circ A$ sensor and Slope $(V)$ is the voltage designating acceleration change per unit length along the model. Theoretically, this approach is independent of the vibration mode or modes. Since the accelerometers are on board the model and being exposed to all vibration modes, the result should be a combined effect and yield an effective $\omega$ and $V$ at any point in time.

\section{Data Analysis}

Wind tunnel model AoA is a difficult quantity to measure. All measurement systems are subject to various biases and errors. Some of the systems suffer from sting whip related problems, some have rectification problems and some have resolutions difficulties and etc. With an absence of an absolute reference for measuring AoA under dynamic conditions, the average of the two video and the two sting whip corrected readings were taken as the reference for each data point.

Bias shifts were tracked at several points during the test using the LaRC Angle Measurement System (AMS) as a reference while performing a wind-off polar. Under these conditions, the AMS is accurate to within $0.002^{\circ}$. The AMS package was attached to the model leveling-plane at specific times during the test and compared to each instrument. This procedure was performed before and after the portion of the test that compared the AoA readings. The before and after biases of each instrument were averaged and the offsets were applied to the data.

The plots in the results section below for AoA verification show the difference between the bias corrected individual instrument measurements and the averaged value. One plot illustrates the severity of the sting whip magnitude by showing the difference between the averaged AoA value and both the sting whip corrected and uncorrected values for QSI and QS2.

Having two sting whip packages in the model located at different fuselage stations made a nice reference for the extrapolation technique. By knowing the correction amount at two different locations and the distance between them, the extrapolation slope can be determined. This was then compared to the calculated extrapolation using the equations above.

\section{$\underline{\text { Results }}$}

The best result to show first is the sting whip magnitude and how well the correction system works. Showing the magnitude is important to the verification because if there is just a low level of sting whip, then we are demonstrating the effectiveness of the AoA sensor instead of the sting whip correction system. The magnitude of the sting whip induced AoA error and the correction capabilities of the sting whip system are shown in Figure 3. This plot shows both QSI and QS2 with the sting whip corrected and uncorrected values. These values are from $M=0.9$ (the mach number that generates the most sting whip error), have been subtracted from the best AoA estimate, and are shown as absolute values for clarity. This plot clearly shows significant error in the uncorrected AoA measurement. The largest error in QS1 is nearly $0.2^{\circ}$ and the largest error in QS2 nearly $0.1^{\circ}$.

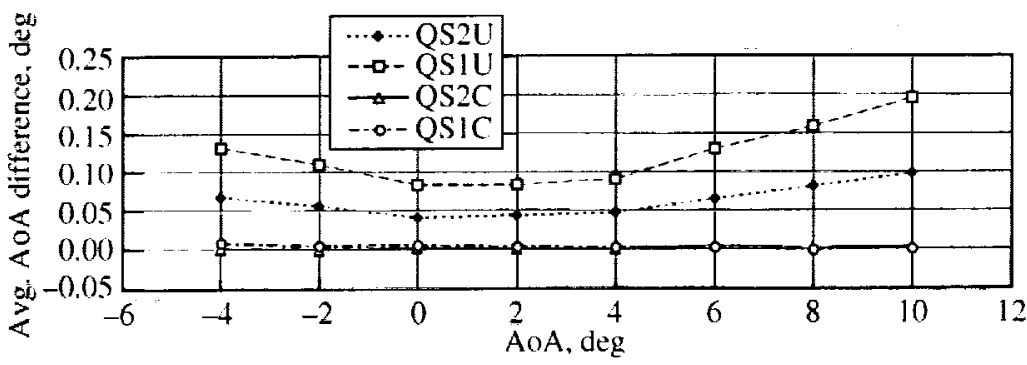

Figure 3. Corrected and uncorrected magnitude January $2001, M=0.9$. 
In all cases the uncorrected values are greater than the required AoA measurement accuracy of $0.01^{\circ}$. For the conrected values, all are within the $0.01^{\circ}$ threshold with the greatest magnitude being $0.0073^{\circ}$ for the QS 1 sensor at the $-4^{\circ}$ alpha position. Although these numbers are generally representative of previous wind tunnel tests, the corrected values at the high alpha positions (high sting whip induced error positions) usually fall within $0.01^{\circ}$.

Another method of showing the accuracy of the sting whip correction system is to plot QS1, QS2, and the two video estimates with the best estimate of alpha subtracted out. This is shown in Figure 4 below.

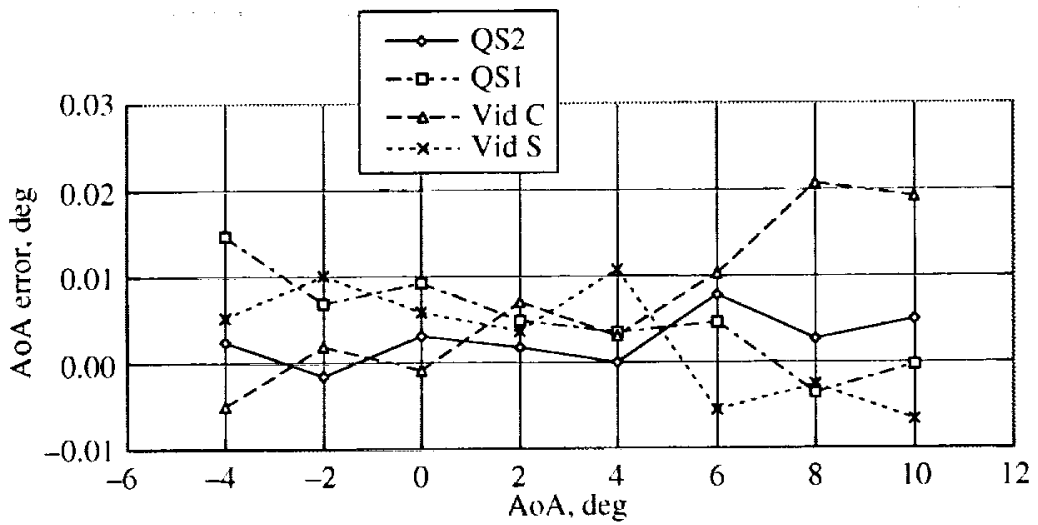

Figure 4. I6-FT TT AoA errors January 2001, $M=0.9$.

In this figure there is good AoA agreement between the video estimates and the sting whip corrected estimates. All of the data points shown are within $0.01^{\circ}$ except for one of the video points.

The results from the extrapolation test are shown in Figure 5. This figure shows that the extrapolation is not quite as good as we had hoped. The extrapolation correction is off by $0.048^{\circ}$ at QS2 and by $0.094^{\circ}$ at the model CG.

Lab results performed on a dynamic rate table showed much better correlation between a secondary accelerometer and the extrapolation from a sting whip package. Investigations are currently under way to determine if simultaneous yaw and pitch motion and if multiple vibration modes are contributing factors. Other potential sources of error are sensor accuracy and the assumption that the model is a rigid body. Any or all of these could contribute to the errors we observed.

Since the AoA data compared favorably, it is reasonable to assume that the correction amounts from QS1 and QS2 are correct. Given this, we feel that the plot of the extrapolation correction line that passes through the QSI and QS2 corrections in Figure 5 is the best. The balance axial correction from this slope calculates to be $0.003 \%$ of full scale (well below the stated accuracy for the axial on this balance of $\pm 0.12 \%$ of full scale). For this model and support system the

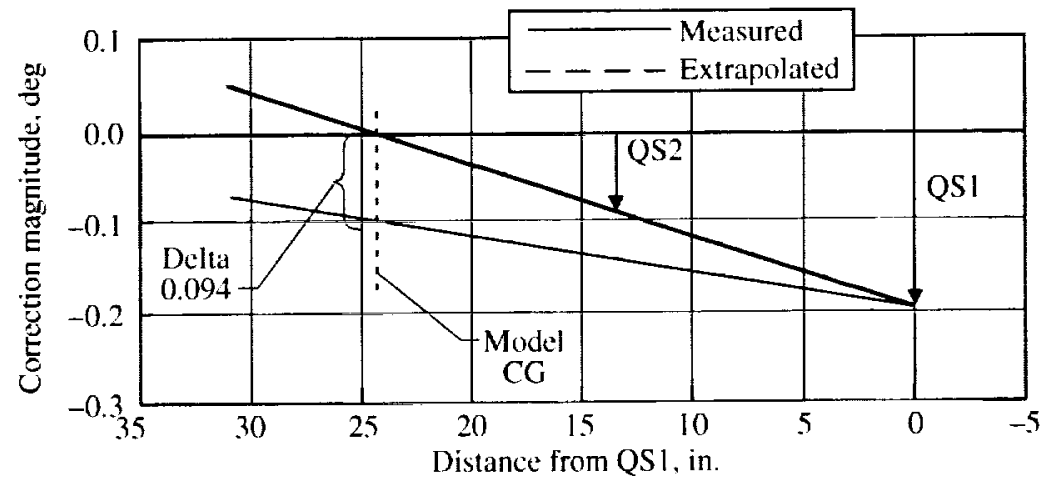

Figure 5. 16-FT TT Measured correction and extrapolation January, 2001, $M=0.9, \mathrm{AoA}=10^{\circ}$. 
axial correction is very small because of the proximity of the vibration node to the model CG. This may not be the case for all models in all tunnels.

\section{Conclusions}

The primary reason for the test was to validate the accuracy of the sting whip correcting AoA measurement system in the presence of a sizable sting whip error. Data presented above clearly shows the ability of the systems to correct for sting whip induced bias errors in the AoA measurement. The systems generally remove $85 \%$ to $90 \%$ of this error, which brings all but the extreme cases to within the $0.01^{\circ}$ accuracy target at LaRC.

Although two sting whip systems will give the necessary information to find the magnitude of the centrifugal acceleration at any point along the model axis, researchers are rarely afforded the luxury of space in the fuselage to accommodate them. In order to get an accurate extrapolation from one sting whip system, more work is needed to determine the error sources.

\section{Latest "Sting Whip" Version}

The latest version of the sting whip instrumentation package reverts from the QS2 technology back to the QS1 technology as described in our earlier paper. ${ }^{4}$ In QSI the (1) term was calculated using the difference of a fore and aft accelerometer and in QS2 it was measured directly using an angular rate sensor. The reason for the change is that the rate sensors were intolerable of the temperatures experienced in many wind tunnels. Failures were experienced in the rate sensors at temperatures as low as $115^{\circ} \mathrm{F}$ while we are required to with-stand temperatures up to $160^{\circ} \mathrm{F}$. It is obvious that a higher temperature device is needed.

The new version is based on QS1 technology in that the $\omega$ term is derived from the difference between the forward and aft accelerometer readings. The difference between the new and the old package is that the new one (QSM) uses MEMS accelerometers as opposed to the piezoelectric accelerometer in QS1.

There are several advantages to the MEMS devices over the piezoelectric. The biggest advantage is the relatively small sensitivity change with temperature change. This improvement is important because of the method of calculating the $\omega$ term. If the sensitivity of the forward sensor changes relative to the rear sensor, then a large error in calculating the $\omega$ term will result.
Having less overall sensitivity shift and pairing sensors with nearly the same shift minimizes this effect.

Some other benefits of the MEMS sensors are: they have static output making them much easier to calibrate, they are considerably cheaper. the signal conditioning is simpler, and they have a higher signal level. There are two main detractions to the MEMS sensors: the need for individual voltage regulators when using a common power feed, and there is no connector on the sensor so the leads have to be manually soldered onto the terminals. The benefits of MEMS far outweigh the detractions.

\section{Acknowledgments}

We would like to thank Ann Bare, Dan Cler, Wes Goodman and all of the tunnel operators from the LaRC/AAAC/ Research Facilities Branch that contributed to the success of this test. It was their willingness to provide us with tunnel time and their cooperation and effort during the test that made this test possible.

We would also like to thank Alpheus Bumer, William Goad and all of the people from the LaRC/AAAC/Instrumentation Systems Development Branch that helped set up and operate the VMD equipment. These are the people that made the comparisons possible.

\section{References}

1. Burner, A.W.; Radeztsky, R.H.; Liu, Tianshu: Videometric Applications in Wind Tunnels. Videometrics $\mathrm{V}$ of the Society of Photo-Optical Instrumentation Engineers, July 30-31, 1997.

2. Mercer, C.E.; Bemier, B.L.; Capone, F.J.; Grayston, A.M.: Data Reduction Formulas for the 16-Foot Transonic Tunnel NASA Langley Research Center Revision 2. NASA-TM 107646, July 1992.

3. Steinle, F.W., Jr.: Investigation of Possible Axial Force Error Induced by Random Oscillatory ModelSupport Motion. Thirty-First Semiannual Meeting of the Supersonic Tunnel Association, April 24-25, 1969 (referenced with permission of the author).

4. Crawford, B.L.; Finley, T.D.: Improved Correction System for Vibration Sensitive Inerial Angle of Attack Measurement Devices. AIAA-2000-0415, Jan. 2000. 